\title{
HIV interactions with herpes viruses in human lyphoid tissues Leonid Margolis*
}

\author{
Address: National Institute of Child Health and Human Development, Bethesda, Maryland, 20892, USA
}

* Corresponding author

from 2006 International Meeting of The Institute of Human Virology Baltimore, USA. 17-2I November, 2006

Published: 2I December 2006

Retrovirology 2006, 3(Suppl I):S63 doi:I0.I I86/I742-4690-3-SI-S63

(C) 2006 Margolis; licensee BioMed Central Ltd.

Critical events in HIV disease occur in lymphoid tissues that are often coinfected with other pathogens, in particular with herpesviruses, which may affect HIV. Here, we investigated infection by and evolution of R5 and X4 HIV1 variants in human lymphoid tissues coinfected ex vivo with human herpesviruses (HHV) including HHV-6, HHV-7 and HCMV. We found that both HHV- 6 and HHV7 selectively suppress $\mathrm{R} 5$ replication, favoring X4 emergence as the dominant phenotype, and investigated the mechanisms of these phenomena. The selective pressure of HHV-7 on HIV-1 is mediated by down-modulation of CD4 in coinfected tissues, whereas HHV-6 mediates its effect on HIV-1 by dramatic upregulation of RANTES. In macaques coinfected with HHV-6 and SIV, HHV-6-resistant escape mutants are selected. These mutants are resistant to RANTES, or even dependent on this chemokine. HIV-1 infection does not affect the replication of HHV-6 but significantly changes the replication of $\mathrm{CMV}$ and HHV-7 depending on HIV-1 phenotype.

Thus, human herpesviruses interact with HIV-1 in coinfected human lymphoid tissue via mechanisms that include modulation of HIV receptors and coreceptors, changing the cytokine/chemokine network, and competing for the same cell targets. Interactions between herpesviruses and HIV might affect the course of HIV disease by selecting for HIV-1 of a particular phenotype as well as for escape mutants. These findings support our hypothesis that interaction of HIV with other microbes in coinfected tissues can determine the pattern of HIV infection. Decipherment of the mechanisms of this phenomenon may significantly contribute to the development of efficient anti-HIV therapies. 